\section{From DIADEM to BigNeuron}

\author{
Hanchuan Peng ${ }^{1} \cdot$ Erik Meijering $^{2}$ - Giorgio A. Ascoli ${ }^{3}$
}

Published online: 29 April 2015

(C) Springer Science+Business Media New York 2015

The three-dimensional (3D) morphology of axons and dendrites is important for many neuroscience studies. Common tasks such as distinguishing and characterizing neuron phenotypes, modeling projection and potential connectivity patterns, and simulating the electrophysiological behavior of single neurons and neuronal networks all depend on accurate knowledge of 3D neuronal morphology. In fact, such tasks often require the morphology to be explicitly and quantitatively described as opposed to simply illustrated by an image stack. ${ }^{1}$ Therefore a critical first step in many studies is the digital reconstruction of the 3D morphology of neurons from image stacks.

Neuron reconstruction methods have evolved over the last 100 years from the 2D hand drawings by Ramón y Cajal and his contemporaries to quantitative tracing of neuron morphologies in 3D with the help of computers. To this day, manual tracing is still the prevailing method

${ }^{1}$ Parekh, R., \& Ascoli, G. A. (2013). Neuronal morphology goes digital: a research hub for cellular and system neuroscience. Neuron, 77(6), 1017-38.

Giorgio A. Ascoli

ascoli@gmu.edu

Hanchuan Peng

hanchuanp@alleninstitute.org

Erik Meijering

meijering@imagescience.org

1 Allen Institute for Brain Science, Seattle, WA, USA

2 Erasmus University Medical Center Rotterdam, Rotterdam, Netherlands

3 Krasnow Institute for Advanced Study, George Mason University, Fairfax, VA, USA even for 3D reconstruction. ${ }^{2}$ However, manual approaches are prohibitively expensive for analyzing image data approaching the scale of terabytes and thousands of image stacks, let alone mining higher-order patterns in these data.

The long-standing need to automate the laborious and subjective manual analysis of light-microscopic and other types of microscopic images has motivated a large number of bioimage informatics efforts. ${ }^{3}$ The recent advance in imaging throughput, combined with the desire for large-scale computational modeling, has added a sense of urgency to this need.

In 2010 a worldwide neuron reconstruction contest named DIADEM (short for "digital reconstruction of axonal and dendritic morphology") was organized by several major institutions as a way to stimulate progress and attract new computational researchers to join the technology development community. ${ }^{4}$ The goal of DIADEM was to develop algorithms capable of automatically converting stacks of images visualizing the tree-like shape of neuronal axons and dendrites into faithful 3D digital reconstructions. The contest succeeded in stimulating a burst of progress. However, none of the algorithms presented at the finishing stage of DIADEM reached the

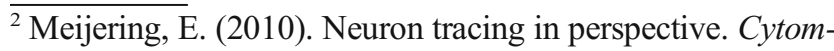
etry Part A, 77(7), 693-704.

${ }^{3}$ Peng, H., Tang, J., Xiao, H., Bria, A., Zhou, J., Butler, V., Zhou, Z., Gonzalez-Bellido, P. T., Oh, S. W., Chen, J., Mitra, A., Tsien, R. W., Zeng, H., Ascoli, G. A., Iannello G., Hawrylycz, M., Myers, E., \& Long, F. (2014). Virtual finger boosts three-dimensional imaging and microsurgery as well as terabyte volume image visualization and analysis. Nature Communications, 5, 4342. doi:10.1038/ncomms5342.

${ }^{4} \mathrm{http}: / /$ diademchallenge.org
} 
originally projected goal of a 20 -fold speed-up in the reconstruction process compared to manual reconstruction. ${ }^{5}$

One practical limitation of DIADEM was that the reconstruction methods were implemented in different languages, ran on different platforms, and followed different protocols to load image data and export reconstructions. This hampered a direct comparison of the methods in terms of computational efficiency and has ever since been a stumbling block to further extend the experiment to big-data, high-throughput applications. In addition, several relatively successful methods recently used in various neuroinformatics projects were introduced ${ }^{6}$ or continued to be developed ${ }^{7}$ after the DIADEM contest.

Current reconstruction techniques, both manual and automated, show tremendous variability in the attributes and completeness of the resulting morphology. ${ }^{8}$ Yet, building a large library of high quality $3 \mathrm{D}$ neuron morphologies is essential to comprehensively cataloging the types of cells in a nervous system. Furthermore, enabling comparisons of neuron morphologies across species will provide additional sources of insight into neural function. It would be beneficial for neuron reconstruction related research and applications to aggregate and consolidate the collective progress on automated neuron tracing in a practically useful product for neuroscience applications. One strategy to overcome the difficulties in dealing with different tracing protocols, data formats, usability, and reproducibility, is to port the available methods to a common, versatile software platform. This allows the methods to be bench-tested against very large-scale neuron datasets for effective validation.

The BigNeuron project has been formally launched in March 2015 to achieve such goals. ${ }^{9}$ This project aims to gather a worldwide community to define and advance the state-of-

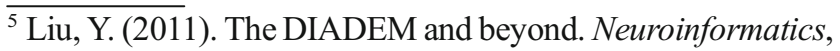
9(2-3), 99-102.

${ }^{6}$ Xiao, H., \& Peng, H. (2013). APP2: automatic tracing of 3D neuron morphology based on hierarchical pruning of a grayweighted image distance-tree. Bioinformatics, 29(11), 144854.

${ }^{7}$ Wang, Y., Narayanaswamy, A., Tsai, C. L., \& Roysam, B. (2011). A broadly applicable 3-D neuron tracing method based on open-curve snake. Neuroinformatics, 9(2-3), 193217.

${ }^{8}$ Parekh, R., Armañanzas, R., \& Ascoli, G. A. (2015). The importance of metadata to assess information content in digital reconstructions of neuronal morphology. Cell Tissue Research, 360(1), 121-7.

${ }^{9} \mathrm{http}: / /$ bigneuron.org the-art of single neuron reconstruction by bench-testing as many automated neuron reconstruction methods as possible against as many neuron datasets as possible following standardized data protocols and evaluation methods. BigNeuron will durably benefit the neuroscience community by establishing a Big Data resource and a set of standardized novel tools for neuron morphologies.

To make BigNeuron a success, tangible goals and feasible approaches have to be developed. While the vision for BigNeuron is to continue for a long time through multiple phases, the first phase will last about a year and a half. The goal of this first phase is to establish the basic infrastructure and release useful data, tools, and analyses. A series of events are being organized for 2015. The kick-off algorithm-porting hackathon was held in Beijing, China in March 2015, with more than 20 attendees from various research groups from Asia, Australia, and America. Follow-up hackathons and workshops will be held at several other locations in Europe and USA. The bench-testing will start in the summer of 2015, followed by data analysis open to the world community. The project welcomes and encourages the participation of any individuals and organizations. Subsequent phases may add important layers of complexity such as time-lapse, multi-channel, and multi-neuron data. In the long run, BigNeuron may also enable a robust cloud-based automated service, where researchers could upload an image stack and receive back a digital morphological tracing.

To advance the neuron reconstruction field, collaborative community projects such as BigNeuron can powerfully complement the competitive spirit of previous initiatives such as DIADEM. The suitability of various neuron reconstruction methods for specific neuron image datasets can still be quantified (and ranked) from the forthcoming BigNeuron results. However, the synergy of many research groups across the globe may provide a refreshing perspective on how various reconstruction methods and results might be integrated. Without a doubt, bench-testing many reconstruction methods against many neuron image datasets will produce very large-scale reconstruction databases. Consensus reconstructions created from all variants generated with each algorithm will also be deposited in NeuroMorpho.Org (ensuring availability in both the de facto standard SWC and NeuroML formats) as well as potentially other databases. Such Big Data will not only benefit method developers and image contributors, but also provide valuable new data resources for computational modelers and data analysts. 